\title{
Starting Current Application for Magnetic Stimulation
}

\author{
Sun-Seob Choi ${ }^{1}$, Gak Hwang Bo ${ }^{2}$, and Whi-Young Kim ${ }^{3 *}$ \\ ${ }^{1}$ Department of Radiology/Neuro Radiology Section, Dong-A University Medical Center, Busan 602-715, Korea \\ ${ }^{2}$ Department Physical Therapy, College of Rehabilitation Science, Daegu University, Gyeongbuk 712-714, Korea \\ ${ }^{3}$ Department of Radiology/Neuro Radiology Section, Dong-A University Medical Center, Busan 602-715, Korea
}

(Received 29 November 2010, Received in final form 12 February 2011, Accepted 14 February 2011)

\begin{abstract}
A power supply for magnetic-stimulation devices was designed via a control algorithm that involved a start current application based on a resonant converter. In this study, a new power supply for magnetic-stimulation devices was designed by controlling the pulse repetition frequency and pulse width. The power density could be controlled using the start-current-compensation and ZCS (zero-current switching) resonant converter. The results revealed a high-repetition-frequency, high-power magnetic-stimulation device. It was found that the stimulation coil current pulse width and that pulse repetition frequency could be controlled within the range of 200-450 $\mu \mathrm{S}$ and 200-900 pps, respectively. The magnetic-stimulation device in this study consisted of a stimulation coil device and a power supply system. The maximum power of the stimulation coil from one discharge was $130 \mathrm{~W}$, which was increased to $260 \mathrm{~W}$ using an additional reciprocating discharge. The output voltage was kept stable in a sinusoidal waveform regardless of the load fluctuations by forming voltage and current control using a deadbeat controller without increasing the current rating at the starting time. This paper describes this magnetic-stimulation device to which the start current was applied.
\end{abstract}

Keywords : magnetic stimulation, half bridge, resonant converter, start current compensation

\section{Introduction}

A magnetic-stimulation device employs electric-field induction in the human body [1]. As the electric field must be tens of volts per meter for electric stimulation, a pulse-type, time-dependent magnetic field of several tesla must be applied to the human body, and the switching time of the time-dependent magnetic field must be under hundreds of milliseconds $[3,4]$. Due to the technical limitations that a strong magnetic field must be switched in a short period of time, the magnetic-field pulse generation frequency of the magnetic-stimulation device is limited to below several hertz at the initial stage. The magnetic-stimulation device has a large stimulation coil capacity because the stimulation frequency must vary when used for different purposes [2].

In addition, attention must be paid to the increase in stimulation coil temperature because the heat generated from stimulation also increases in proportion to the frequency [5]. Magnetic stimulation requires a capacitor

*Corresponding author: Tel: +82-51-200-3449

Fax: +82-51-200-3235, e-mail: ndyag@dongju.ac.kr that stores energy up to several kilo-volts, and a stimulation coil that forms a magnetic field through discharge $[6,7]$. The capacitor generates a several-kilo-ampere current by discharging the charged energy into the stimulation coil within a short period of time (hundreds of seconds). It forms a magnetic field in pulse form and generates an eddy current in the human body [8]. This performance requires a large-capacity power supply, high-voltage resistance, high repetition, high-speed switching element, and diverse control techniques [9].

A general magnetic-stimulation device boosts then rectifies the sinusoidal power from a commercial power source (AC $220 \mathrm{~V}$ and $60 \mathrm{~Hz}$ ) to charge the capacitor at a high voltage [7]. This has problems in terms of the transformer turn ratio, power loss, size, system complexity, and high voltage and current control [8]. To solve these problems, the magnetic-stimulation device should have high power density and diverse controls according to the diagnostic purpose. The power density can be regulated by controlling the operating time using the pulsed magnetic-stimulation device output, and by changing the pulse repetition frequency.

For this purpose, the ZCS resonant converter type was 
used to examine the electrical and output characteristics of the magnetic-stimulation device while varying the current pulse width and pulse repetition frequency. As a result, a reverse-direction circuit was added to the existing device, in which the pulse was outputted from the stimulation coil once, resulting in one more output from the stimulation coil.

In addition, a deadbeat control was used to control the voltage and current to compensate for the dynamic characteristics due to load fluctuations and flux imbalance of the inductor. The output voltage was controlled in constant current mode using a control algorithm, in which the output voltage could be kept constantly sinusoidal regardless of the load fluctuations, and using an added control mode when a current higher than the rated current was required.

\section{Circuit Topology and Operational Principle}

The magnetic-stimulation device is suitable for delicate diagnoses because its current pulse control is reliable and it generates high-quality magnetic pulses. It is currently the flow in the diagnosis and treatment of cranial nerve or muscular diseases. It mostly generates sinusoidal waves because it is employed for diagnosis and treatment, but it requires a wide range of magnetic-pulse output densities. In addition, a magnetic-stimulation device with multipleenergy pulses allows deeper penetration with better results than a single-energy pulse at the same average output. The power supply manufactured in this study is suitable for high frequencies and has reduced switching loss and noise. In particular, a ZCS (zero cross-switching) resonant converter was applied to the magnetic-stimulation device to reduce the losses due to the tailing current when the IGBT was turned off, enabling output control according to the frequency control within a specific pulse width.

Fig. 1 shows a circuit with a half bridge, start-currentcompensation circuit, and ZCS resonant converter (pulseforming network or PFN). As an excessive peak current is outputted from the main circuit of the start-currentcompensation-type ZCS serial resonant converter, a largeoutput DC capacitor with excellent frequency characteristics that is connected parallel to a smoothing condenser was selected. Fig. 1 shows the operation principle of the circuits. For applications of the ZCS serial resonant converter, the PFN consisted of switching elements (S1 and S2), resonant inductor (L1), capacitors (c1 and c2), and a start current element (S3). This theoretically involves no switching loss and is suitable for high-repetition-frequency operation because it switches the switching elements on or off at zero current when a sinusoidal current flows through the switching element and capacitor. Equation 1 shows the output characteristics of the start-current-compensationtype ZCS serial resonant converter.

$$
\text { Pout }=2 f c f V \text { in }^{2} \text {. }
$$

The power density can be controlled using two methods: varying the input voltage of the start-current-compensationtype resonant converter within a specific pulse width and frequency, and varying the switching repetition frequency of the resonant converter. The method of varying the input voltage was applied because repetition frequency control would have caused instability and noise from low-

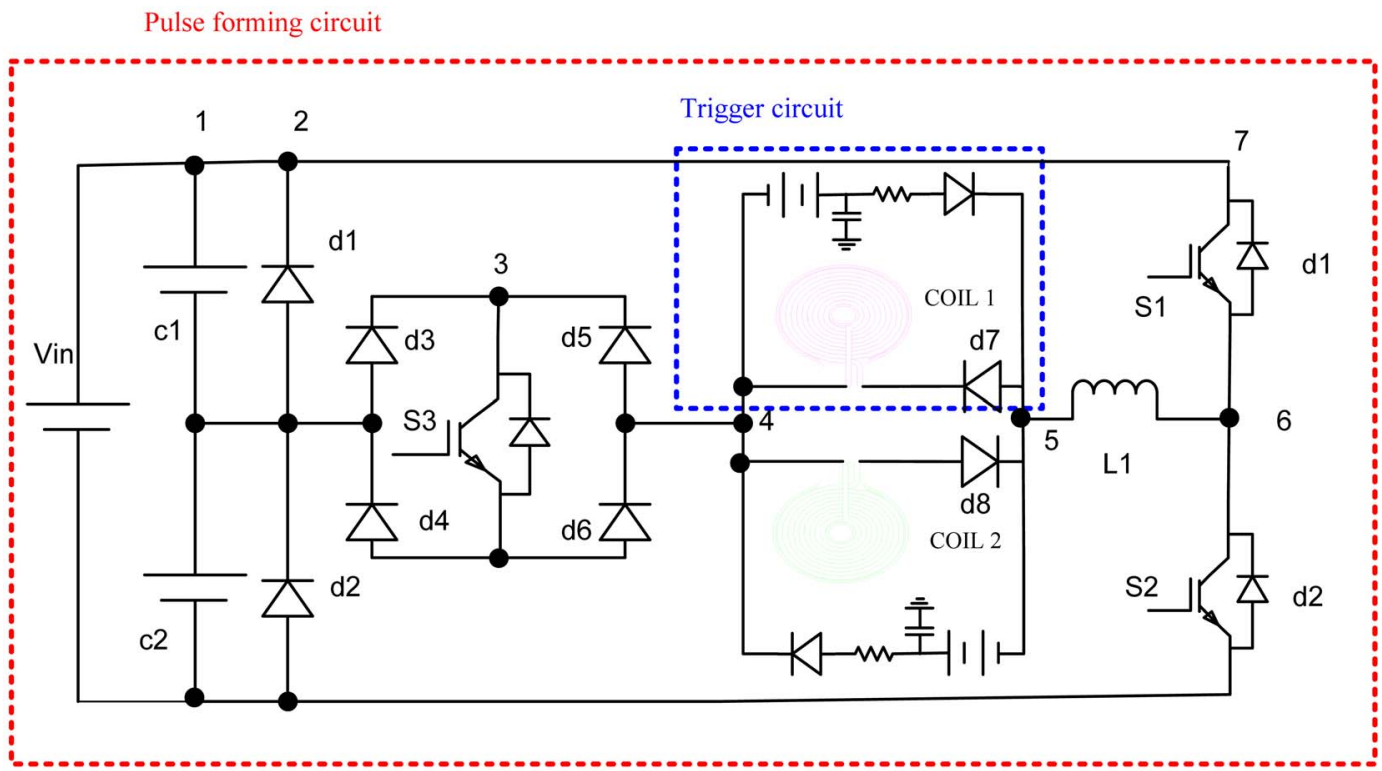

Fig. 1. Proposed magnetic-stimulation device circuit. 
frequency switching when the frequency decreased under a light load. Initially, $\mathrm{c} 1$ is charged with an input voltage Vin, c2 has a zero voltage, and S1 and S2 do not allow current to flow through the stimulation coil. When S1 is switched on, the resonant current will flow through loops $\mathrm{c} 1 \rightarrow \mathrm{S} 1 \rightarrow \mathrm{L} 1 \rightarrow \mathrm{d} 7 \rightarrow$ coil $1(\mathrm{~F}) \rightarrow \mathrm{d} 5 \rightarrow \mathrm{S} 3 \rightarrow \mathrm{d} 4 \rightarrow$ $\mathrm{c} 1$ and $\mathrm{Vin} \rightarrow \mathrm{S} 1 \rightarrow \mathrm{L} 1 \rightarrow \mathrm{d} 7 \rightarrow$ coil $1 \rightarrow \mathrm{d} 5 \rightarrow \mathrm{S} 3 \rightarrow$ $\mathrm{d} 4 \rightarrow \mathrm{c} 1$, and will supply energy to coil 1 (forward).

After $\mathrm{c} 1$ is discharged at time $\mathrm{t} 1$, the current in resonant inductor L1 will flow through loop L1 $\rightarrow$ d7 $\rightarrow$ coil 1 (F) $\rightarrow \mathrm{d} 1 \rightarrow \mathrm{S} 1 \rightarrow \mathrm{L} 1$ and will clamp the voltage of resonant capacitor $\mathrm{c} 1 \mathrm{( \textrm {Vc } 1 )}$ with a zero voltage and the voltage of resonant capacitor $\mathrm{c} 2$ with the input voltage, Vin.

It is best that $\mathrm{S} 1$ be switched off after the resonant current reaches zero but the tailing current continues to flow due to the characteristics of coil 1 . Therefore, if it is forcibly turned off after the calculated on-time, the resonant current will flow through loop L1 $\rightarrow$ d7 $\rightarrow$ coil $1(\mathrm{~F}) \rightarrow$ $\mathrm{d} 5 \rightarrow \mathrm{S} 3 \rightarrow \mathrm{d} 4 \rightarrow 1 \rightarrow \mathrm{Vin} \rightarrow \mathrm{s} 2 \mathrm{D} \rightarrow$ L1 to reduce the resonant current to zero. At that time, no current will flow through coil 2 because it is blocked by $\mathrm{d} 8$, and the energy will be supplied only to coil 1 . When $\mathrm{S} 2$ is switched on after a specific time, the resonant current will flow through loops $\mathrm{c} 2 \rightarrow \mathrm{d} 3 \rightarrow \mathrm{S} 3 \rightarrow \mathrm{d} 6 \rightarrow$ coil $2 \rightarrow \mathrm{d} 8 \rightarrow \mathrm{L} 1 \rightarrow \mathrm{S} 2$ $\rightarrow \mathrm{c} 2$ and $\mathrm{Vin} \rightarrow \mathrm{c} 1 \rightarrow \mathrm{d} 3 \rightarrow \mathrm{S} 3 \rightarrow \mathrm{d} 6 \rightarrow \operatorname{coil} 2 \rightarrow \mathrm{de} 8$ $\rightarrow \mathrm{S} 2 \rightarrow$ Vin. The inverse resonant current will flow through L1 to supply energy to coil 2 . After c2 is discharged, the resonant current of L1 will flow through loop $\mathrm{L} 1 \rightarrow \mathrm{s} 2 \rightarrow \mathrm{d} 2 \rightarrow \mathrm{d} 3 \rightarrow \mathrm{S} 3 \rightarrow \mathrm{d} 6 \rightarrow$ coil $2 \rightarrow \mathrm{d} 8 \rightarrow \mathrm{L} 1$ to emit energy to coil 2 , and the current will become zero. The voltage between both ends of resonant capacitor $\mathrm{c} 1$ (Vc1) will be clamped with Vin due to $d 2$, and the subsequent sequence will be repeated. To compensate for the dynamic characteristics due to the load fluctuation and flux imbalance of the inductor, a deadbeat control was used to control the voltage and current. Equation 1 shows the linear model in Fig. 1, where $r L$ is the equivalent serial circuit of the inductor, $r C$ is the equivalent serial circuit of the capacitor, and $R$ is the load resistance.

$$
\begin{aligned}
& {\left[\begin{array}{l}
i L \\
V c
\end{array}\right]=\left[\frac{\frac{r L r c+R(r L+r c)}{L(R+r c)} \frac{-R}{\frac{L(R+r c)}{c(R+r c)}}}{\frac{-1}{c(R+r c)}}\right]\left[\begin{array}{c}
i L \\
V C
\end{array}\right]+\left[\begin{array}{c}
i / L \\
0
\end{array}\right] V i,} \\
& v o=\left[\frac{\frac{R r c}{(R+r c)}}{\frac{(R}{(R+r c)}}\right]\left[\begin{array}{l}
i L \\
v C
\end{array}\right] .
\end{aligned}
$$

Fig. 2 shows the current control mode. Equation (4) shows the output of the reference inductor voltage in terms of the feed forward control.

$$
v L(k)=K_{P}[i L(K)-i L(K)]+v o(k) .
$$

Equation (5) shows the transfer function of the zeroorder holder.

$$
G_{k}(S)=\frac{1-e^{-S T}}{S} .
$$

Equation (6) shows the transfer function of the inductor.

$$
G_{k}(S)=\frac{1 / L}{s+a} \text {, and } a=\frac{r L}{L} \text {. }
$$

Therefore, equation (7) is the open-loop transfer function.

$$
G_{s}(S)=\frac{1}{r L} \frac{1-e^{a T}}{1-e^{a T}} .
$$

The characteristic equation of the closed loop can be expressed as

$$
z-\left[e^{a T}-K_{p} \frac{e^{a T}-1}{r L}\right]=0 .
$$

$k_{p}$ can be calculated with the pole placed at the origin for the dynamic characteristic of the current control mode

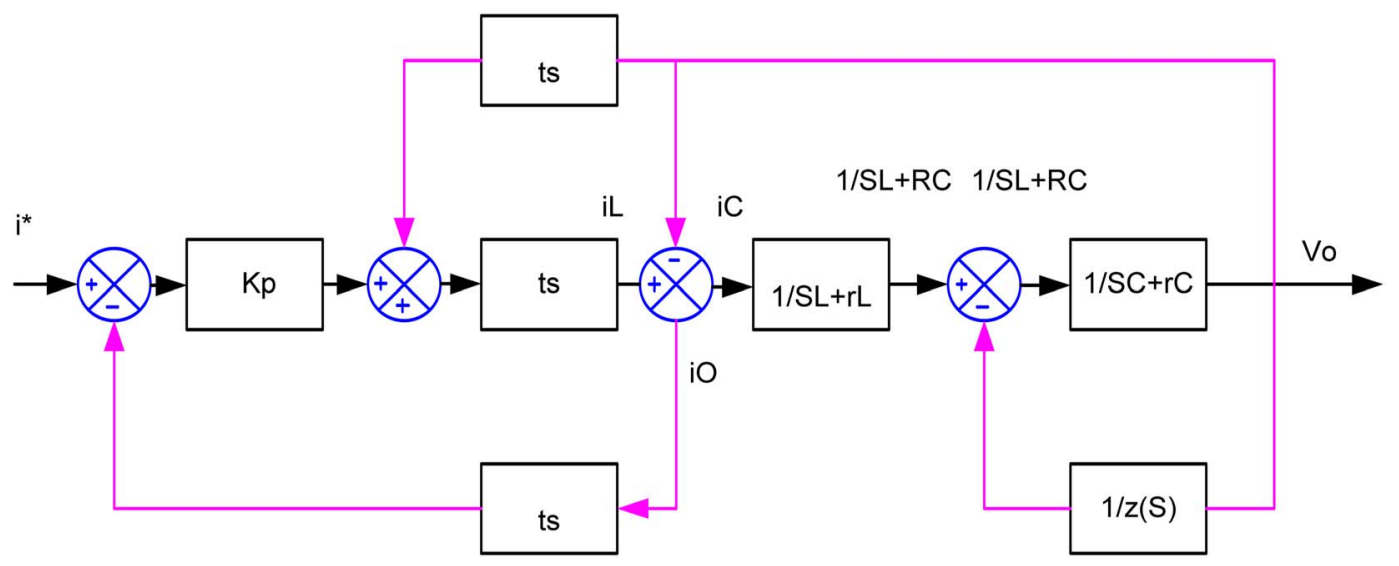

Fig. 2. Current control mode. 


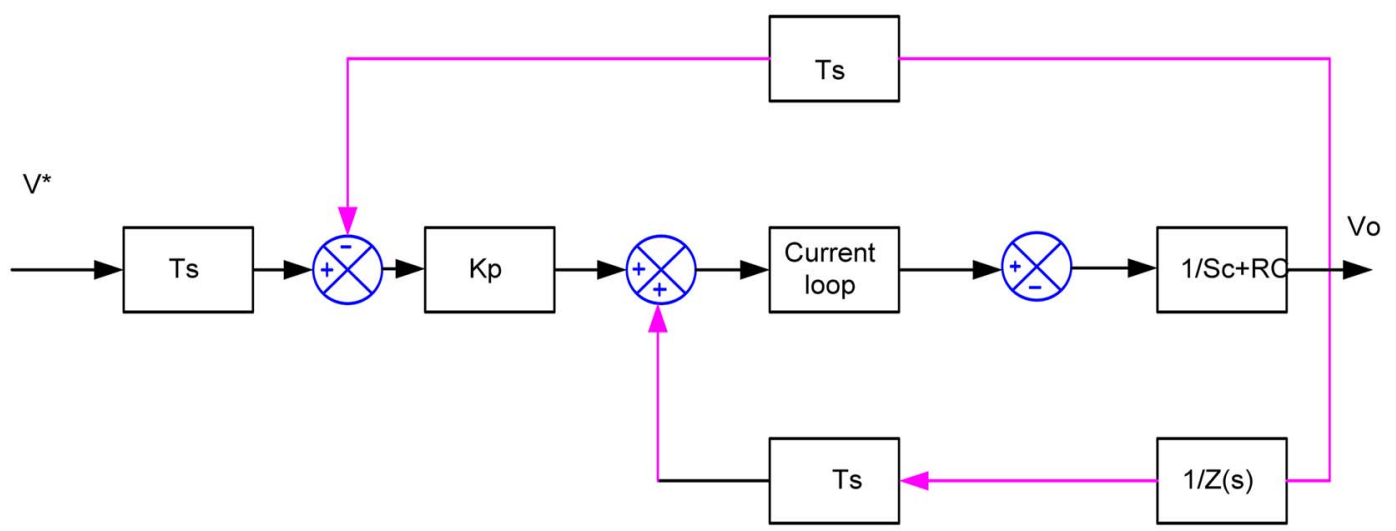

Fig. 3. Voltage control mode (including a current controller in the inner loop).

(equation 9).

$$
r_{p}=\frac{r L e^{-a T}}{1-e^{a T}} .
$$

Fig. 3 shows a block diagram of the voltage control mode. The inner loop of the voltage control mode includes a current controller.

$$
i(k)=K v\left[\operatorname{vref}(k)-v_{0}(k)\right]+i_{0}(k) .
$$

When starting the magnetic-stimulation device, a high current rating is needed to cover a high instantaneous start current. As the current exceeds the rated current, the system is stopped by the fault signal of the overcurrent protector and cannot supply power to the load. The shorttime current rating is determined by the current rating of the switching element. However, the switching element can be broken if the load requires a large current that exceeds the current rating of the switching element. Therefore, the instantaneous current may not exceed the current rating. In this method, the system operates in a rated-current control mode without being stopped, even though a high instantaneous current is needed.

\section{Simulation and Experiment Results}

The driving pulse and display for driving S1, S2, and S3 are controlled using AT90S8535 (ATMEL system) (Fig. 4). AVR (ATMEL90S8535) supplies a wide range of pulses to the IGBT-exclusive driving module EXB841 (FUJI), including delay and overlap, via a key pad. A range of data can be inputted at any time where necessary using a key pad. Fig. 5 shows the existing half-bridge circuit composition and simulation waveforms. Fig. 6 presents the half-bridge circuit composition and simulation waveforms with the start current application. As shown in the results, the half-bridge type generates unstable waveforms while the proposed type results in stable waveforms. When the inductor current exceeds the set value, with the rated

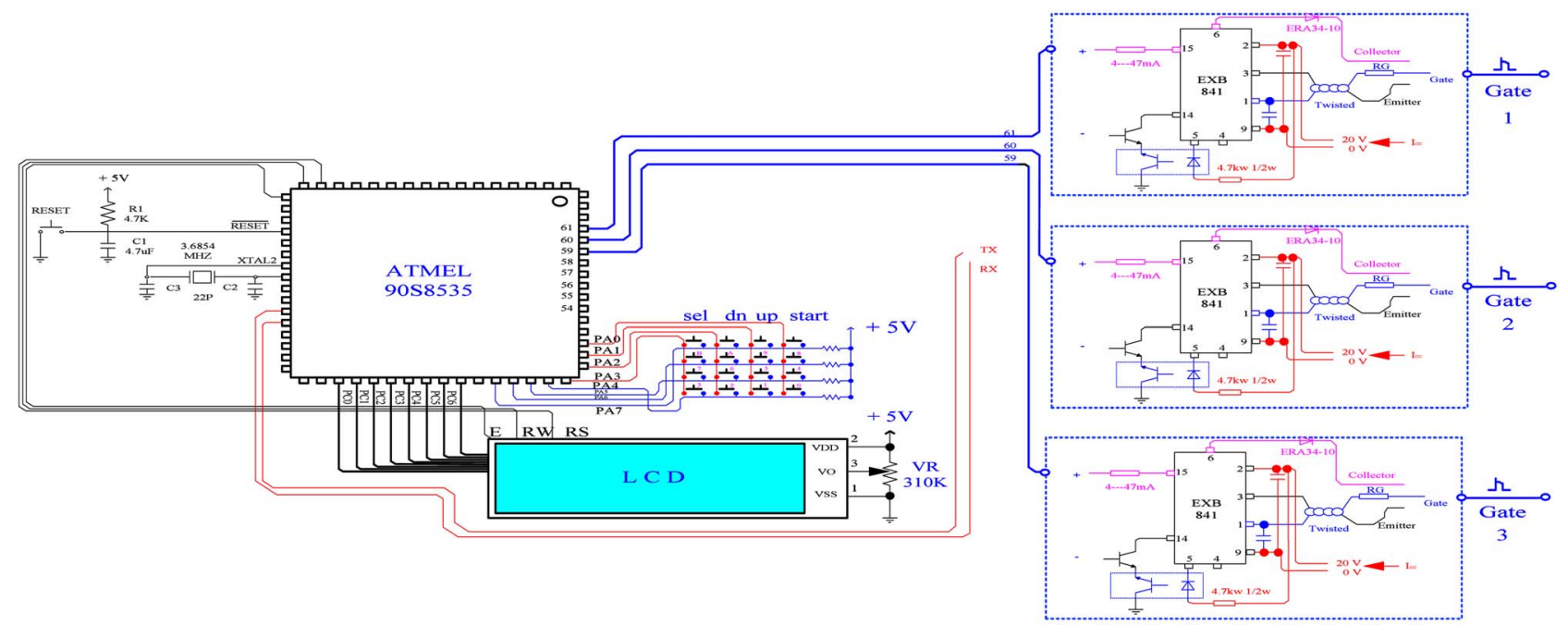

Fig. 4. Driving and control circuit (ATMEL 90S8535). 


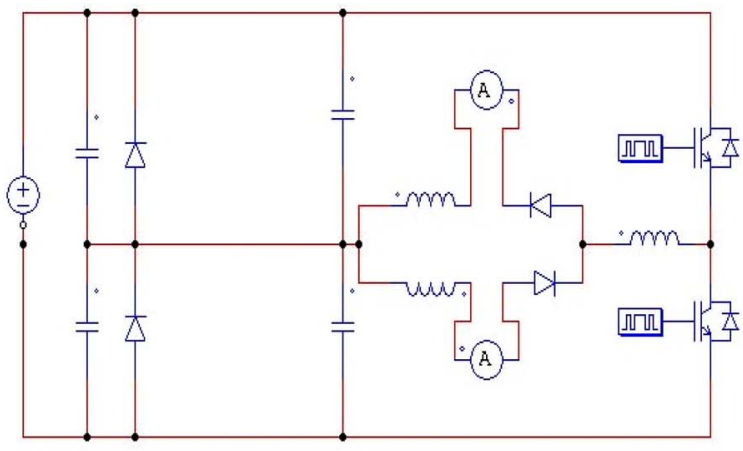

(a) Conventional ZCS resonant converter type

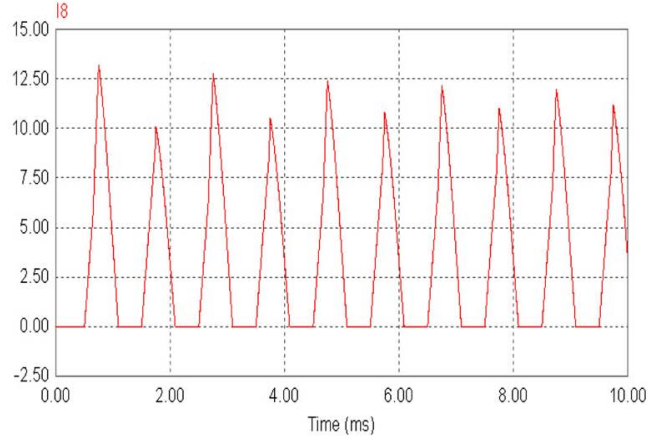

(b) Simulation results

Fig. 5. Conventional half-bridge-type ZCS mode.

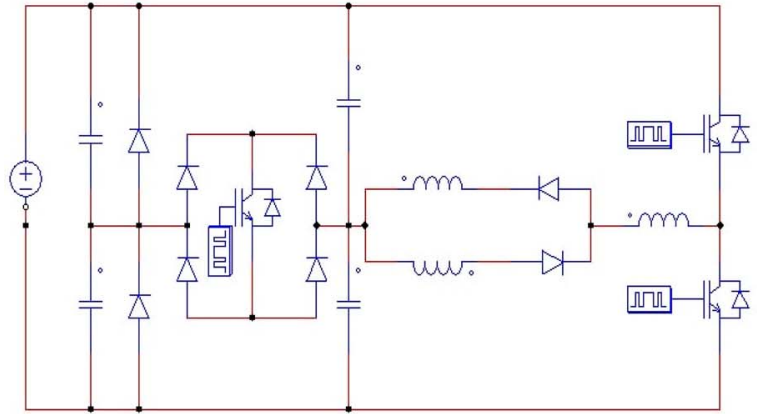

(a) Start-current-compensation ZCS resonant converter type

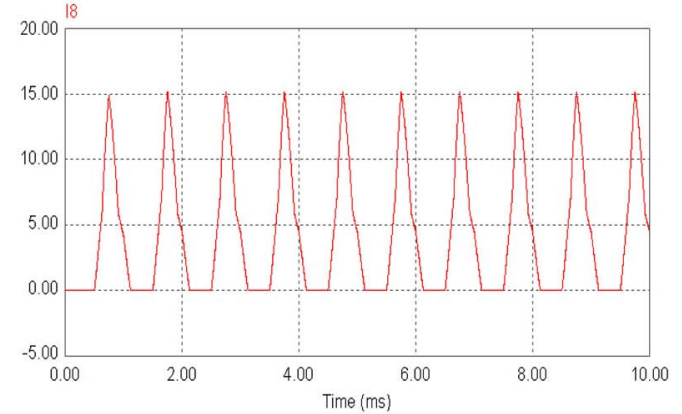

(b) Simulation results

Fig. 6. Proposed ZCS mode of the start-current-compensation half-bridge type.

current exceeded, the current assumes a constant form due to the constant-current control mode, and a slight distortion in the output voltage appears. A stable output voltage will be generated if the load changes to ensure that the current is below the constant-current value. Fig. 7(a) gives the inductor current, load current, condenser

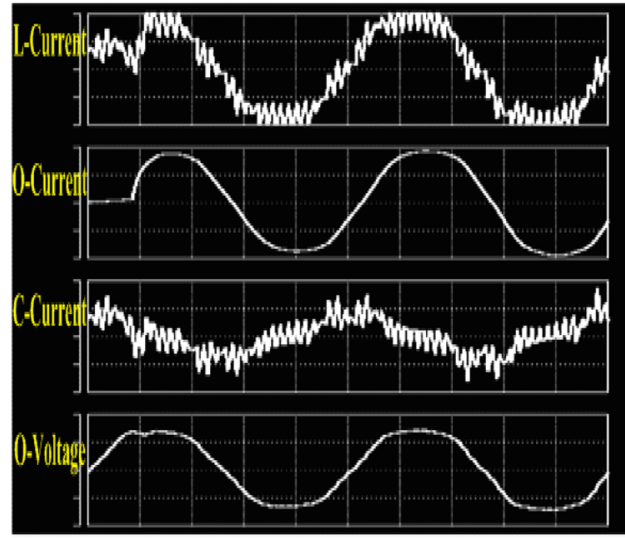

(a) current and output voltage when the driving current changes from below the rated current to above it. Fig. 7(b) presents the inductor current, load current, condenser current, and output voltage when the driving current changes from above the rated current to below it.

Visual $\mathrm{C}++[\mathrm{VC}]$ to implement a monitoring program

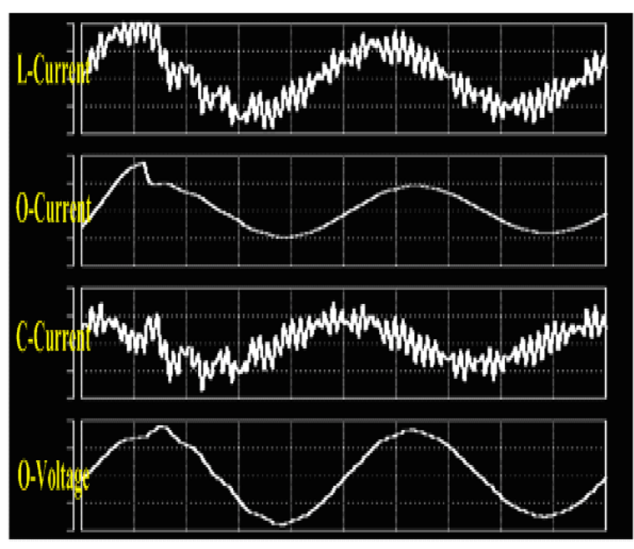

(b)

Fig. 7. (a) When a change in load current from below the rated current to above it is needed. (b) When a change in load current from below the rated current to above it is needed. 
was run. Assuming that no loss from the resonance or discharge circuit, there will be no change in $\mathrm{VC}$ at the start and end of the discharge. On the other hand, the loss appears to be due to the electric resistance of the magneticfield generation coil and wiring, which decreases the discharge completion voltage. With this voltage drop, the power loss of the discharge circuit can be calculated using the following equation. However, the power capacity of the high-voltage power that charges the discharge capacitor must be higher than the power loss. In the experiment, a $220 \mathrm{~V} \mathrm{AC}(60 \mathrm{~Hz})$ source was rectified, and the pulsating current was used as the input power. C was $100 \mu \mathrm{F}$ and $2000 \mathrm{~V}$, and L was manufactured with a $50-\mu \mathrm{H}$ magneticfield generation coil. A half-bridge-exclusive module (3300 $\mathrm{V}, 400 \mathrm{~A}$, DYNEX) was used as the IGBT for the discharge operation, and components with sufficient current capacity and withstand voltage were utilized for the high-voltage, high-current circuit wiring.

Fig. 8 shows the relationship between the repetition frequency and stimulation coil output. Fig. 9 presents the relationship between the charging voltage and joule. The current and voltage were measured using a high-voltage probe (Lecroy, Probe X, 6000 V) and a Rogoski current

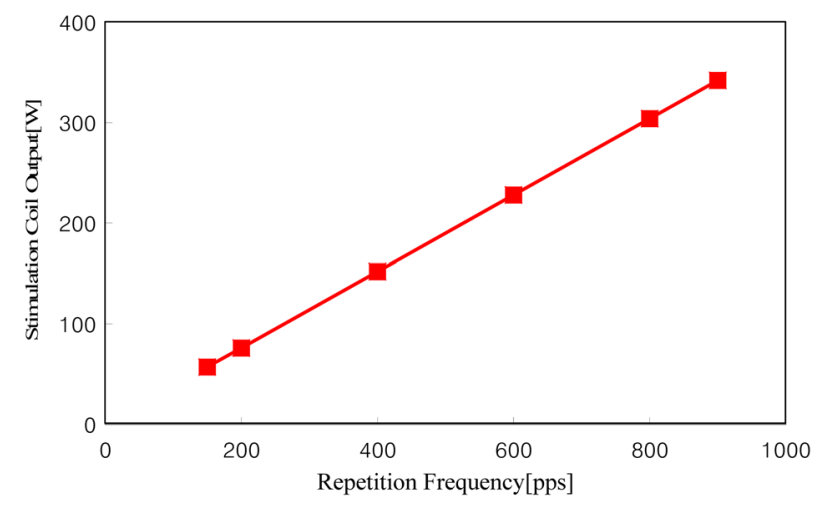

Fig. 8. Output characteristic with reference to the repetition frequency.

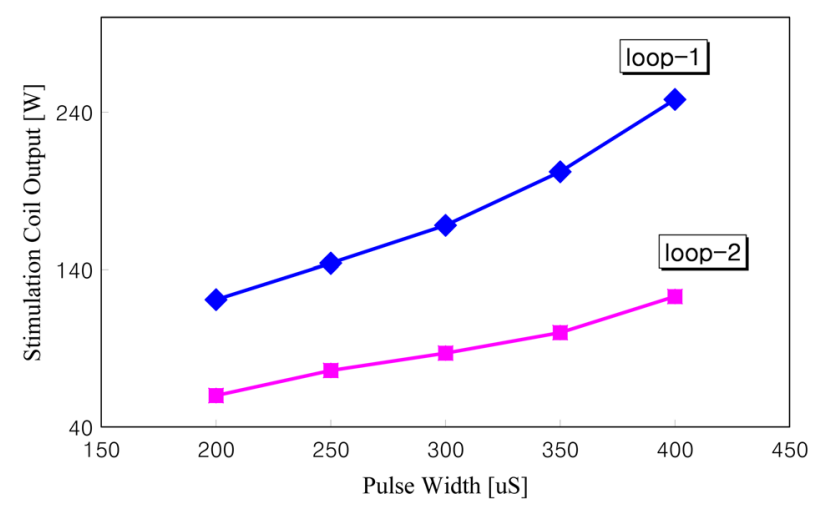

Fig. 9. Joule with reference to the charging voltage $[\mathrm{J}]$. waveform transducer (CWT, Penmuk). The initial-discharge voltage $\mathrm{Vi}$ was $1000 \mathrm{~V}$ and the discharge completion voltage Ve was $760 \mathrm{~V}$. Therefore, the power loss can be calculated using the following equation:

$$
P_{\text {loss }}=\frac{1}{2} C f\left[\left(v_{\text {ini }}\right)^{2}-\left(v_{\text {end }}\right)^{2}\right] .
$$

Within $20 \mathrm{~ms}$ after discharge completion, charging is possible with an initial-discharge voltage (Vi) of $1000 \mathrm{~V}$. A $1000 \mathrm{~V} v_{i n i}$ can realize a stimulation frequency of 50 Hz. When Vi was $1000 \mathrm{~V}$, a time-dependent magnetic field of $1 \mathrm{~T}$ appeared at a point $50 \mathrm{~mm}$ from the stimulation coil. Even when IGBT was switched on during the discharge operation, there was no voltage or current that exceeded the ratings of the components around the IGBT.

\section{Conclusion}

Charging was carried out once when the switching frequency was $40 \mathrm{~Hz}$, and 53 times when the switching frequency of the IGBT was $2 \mathrm{kHz}$. Therefore, the change in the initial charging voltage due to IGBT switching was $3 \mathrm{~V}$ at best according to the phase angle of the $\mathrm{AC}$ power, and only the commercial power was insufficient. The pure half-bridge type was also insufficient in terms of a stable output. In the ZCS resonant inverter in this study, precision control was realized by varying the pulse width and repetition frequency of the current against the magnetic-stimulation device output according to the power density and diagnostic purpose, utilizing the start-currentcompensation type with an IGBT element module.

A new current control method that can control the converter output voltage without increasing the current rating at the start time was introduced, in which the change in control mode and flux imbalance were compensated for. As a result, the output voltage could be kept constant in a sinusoidal manner regardless of the stimulation coil output and load fluctuation. In addition, the proposed device was quite efficient as a high-repetition-frequency and highpower magnetic-stimulation device because it controls the pulse duty ratio of the gate of the IGBT according to the conditions. The magnetic-stimulation device is currently being applied in many fields, and satisfactory results have been obtained via its laboratory design and manufacture in this study.

The operational characteristics of the prototype led to an output power of $130 \mathrm{~W}$, and the serial connection of two devices resulted in a maximum average output of 260 W. A proportional output can be obtained if stimulation coils are added in series with the subsequent changes in the repetition frequency of the output. Using this technique, 
a large-power, high-repetition-frequency, stable magneticstimulation device system, where the energy density of the stimulation device can be precisely controlled, was realized.

\section{Acknowledgement}

This work was supported by the Dong-A University Research fund.

\section{References}

[1] W. Paulus, "Transcranial magnetic stimulation and transcranial direct current stimulation", Proceedings of the $2^{\text {nd }}$ international TMS and tDCS Symposium (2003).

[2] Sun-Seob Choi, J. Magnetics 15, 213 (2010).
[3] C. Plewnia, M. Bartels, L. Cohen, and C. Gerloff, Neurosci. Lett. 307, 41 (2001).

[4] A. Gadea and A. M. Lopez-Colome, J Neurosci Res. 64, 218 (2001).

[5] K.-H. Hsu, Ph.D. dissertation, Dept. Biomed. Eng., Case Western Reserve Univ., Cleveland, OH (2000).

[6] C. Plewnia, M. Bartels, L. Cohen, and C. Gerloff, Neurosci Lett. 307, 41 (2001).

[7] H. Théoret, M. Kobayashi, A. Valero-Cabré, and A. Pascual-Leone, Supplements to Clinical Neurophysiology 56, 211 (2003).

[8] S. Mark and M. D. George, Transcranial Magnetic Stimulation in Clinical Psychiatry, American Psychiatric Publishing, Inc. Arlington, VA (2006).

[9] M. Inghilleri, A. Berardelli, P. Marchetti, and M. Manfredi, Exp. Brain. Res. 109, 467 (1996). 